\title{
Journal highlight: an overview on interesting articles
}

\author{
Nooshin Bagherani ${ }^{1 *}$ and Bruce R Smoller ${ }^{2}$ \\ ${ }^{1}$ MD, Dermatologist at Dr. Nooshin Bagheran's office, Taha Physicians' building, P.O.Box: 6414715878, Khoramshahr, Khuzestan Province, Iran \\ ${ }^{2} \mathrm{MD}$, Professor and Chair, Department of Pathology, Professor, Department of Dermatology, University of Rochester, School of Medicine and Dentistry, USA
}

\section{Role of lasers in treatment of connective tissue diseases}

Connective tissue diseases (CTD) is a group of systemic disorders, characterized by clinically unique skin manifestations often resistant to conventional therapies [1]. In this article, Brauer et al have reviewed the role of lasers in treatment of these manifestations. By searching MEDLINE, they found 39 related articles.

Lupus erythematosus (LE): Its skin manifestations include in acute and chronic cutaneous lupus, oral and nasal ulcers, and non-scarring alopecia. The efficacy of pulsed dye laser (PDL) in treating all kinds of cutaneous LE has been revealed in 8 articles. The relative efficacy of argon laser in improving discoid LE (DLE) has been reported in 2 articles. Successful improvement of DLE with prolonged remission has been reported with ablative carbon dioxide $\left(\mathrm{CO}_{2}\right)$ and ablative yttrium aluminum garnet (YAG) lasers.

Scleroderma and morphea: Skin manifestations of scleroderma are summarized in skin fibrosis, Raynaud's phenomenon, and telangiectasias. The efficacy of PDL in treating telangiectasias has been revealed, but about en coup de sabre and plaque morphea, the results have been variable. Intense pulsed light (IPL) has successfully been used for treating microstomia. The efficacy of ablative and fractional ablative $\mathrm{CO}_{2}$ lasers has been demonstrated in improving rhytides, calcinosis of the digits, and joint contractures. Additionally, the efficacies of excimer and 1064-nm neodymium-doped YAG (Nd-YAG) have been reported in treating morphea lesions and severe Raynaud's disease, respectively.

Sarcoidosis: Regarding existence of non-caseating granulomas, its skin manifestations are classified into specific and non-specific lesions. PDL has successfully been used in treating cutaneous sarcoidosis. In remodeling of lupus pernio, $\mathrm{CO}_{2}$ laser has provided positive responses. However, among the CTDs, sarcoidosis has had the most adverse events from laser therapy.

Dermatomyositis: Its skin lesions include calcinosis, poikiloderma, Gottron's papules, periungual telangiectasias, heliotrope eruption, shawl and holster signs, and mechanic's hands. The efficacy of PDL and argon laser have been reported in improving telangiectasias, poikiloderma, and Gottron's papules.

Commentary: Lasers can find proper placement in the treatment of skin manifestations of CTDs.

\section{Therapeutic options for atrophic acne scarring}

Acne vulgaris is the most common skin disease among adolescents [2]. Atrophic scars, as its common sequel, result in significant psychological distress. They are subdivided into boxcar, icepick, and rolling scars. In this article, Hession and Graber reviewed options for treating atrophic acne scars.
Dermabrasion: It is an operator-dependent technique, which is helpful in removing sharper scar edges. Because of its poor safety profile, it is an inferior choice.

Subcision: It is an effective treatment for rolling scars. In this technique, the bleeding and subsequent clot formation result in elevation of the skin from the underlying scar tissue, which induces neocollagenesis. Its combination with other modalities is more effective than subcision alone. Subcision by loosening deep fibrous attachments can make acne scars more amenable to other treatment modalities.

Skin needling: This technique, as a collagen induction therapy, is effective in treating acne scarring by promoting neocollagenesis in a manner analogous to subcision and fractional ablative lasers.

Punch techniques: These techniques include punch excision, elevation, and grafting which are suitable for correcting icepick scars.

Chemical peeling: A variety of studies support the use of chemical peels in treating acne scars. The efficacy of a new technique of chemical peeling, so-called chemical reconstruction of skin scars, has been shown for treating icepick scars.

Tissue augmentation: In addition to replacing tissue volume, this modality can stimulate collagen production. It is appropriate for large rolling scars.

Fat transfer: This technique is effective for treating acne scars, but its result is highly operator- dependent and short-lived.

Autologous fibroblast transfer: It offers permanent results in treating acne scars.

Laser resurfacing: Over the past decade, it has become the first choice in treating all the types of atrophic acne scars.

Commentary: There are numerous therapeutic options for acne scars based on their types. To achieve maximum results, several modalities can be combined.

\section{Efficacy of podophyllin in treatment of keratoacanthoma}

Keratoacanthoma (KA) is a common skin tumor particularly seen in immune-compromised patients and cases with xeroderma pigmentosa, and xerodermoid types [3]. Because of its rapidly-growing manner, the KA can cause local severe destruction of tissues; hence

Correspondence to: Nooshin Bagherani, MD, Dermatologist at Dr. Nooshin Bagheran's office, Taha Physicians' building, P.O.Box: 6414715878, Khoramshahr, Khuzestan Province, Iran; Email: nooshinbagherani@yahoo.com

Received: September 10, 2016; Accepted: October 19, 2016; Published: October 21,2016 
its treatment especially in early stages is important. Classically, the curettage, cautery, and surgical excision are standard modalities for the treatment of KA. Other therapeutic options include topical immiquimod, topical 5-florouracil, and photodynamic therapy with topical 8-aminolaevullinic acid.

Podophyllin is an antimitotic, cytotoxic, and caustic agent. It can cross cell membranes, and inhibit cell mitosis and DNA synthesis through binding to tubulin. It also prevents polymerization of tubulin into microtubules, resulting in arrest in cell division. Furthermore, podophyllin can block oxidation enzymes in tricaboxylic acid cycle, interfere with nutrition of cells, and inhibit axonal transport, protein, RNA, and DNA synthesis. It also inhibits mitochondrial activity and reduces cytochrome oxidase activity. This toxic agent can induce local necrosis and death of tumor cells.

In previous studies, the efficacy of podophyllin has been shown in treating genital wart, cutaneous leishmaniasis, basal cell carcinoma, and solar keratosis. In the current study, Sharquie and Noaimi introduced podophyllin as an effective and safe therapeutic option for KA. They recruited three cases with KA (2 female xerodemoid patients and 1 male non-xerodermoid patient). Topical $25 \%$ podophylline solution was administered once weekly for maximum of 6 weeks. The patients were followed up for18 months.

Sharquie and Noaimi showed that podophylline was very effective for treating KA with good cosmetic results. Complications or side effects were not reported in this study. Eventually, they concluded that topical podophyllin can be an appropriate option for the treatment of $\mathrm{KA}$ in patients refusing surgical approaches.

Commentary: Podophylline can be an effective and safe agent for the treatment of keratoacanthoma.

\section{Mechanisms underlying aggressive manner of mela- noma}

Melanoma has an aggressive manner, with metastasizing to multiple organs including lung, liver, brain, bone, and lymph nodes [4]. In this article, Braeuer et al have comprehensively reviewed the probable mechanisms underlying this manner:

Antigenicity: Melanoma cells are highly antigenic and share most of their antigens with vascular cells. These antigens allow melanoma cells to adhere to the vessel wall in distant organs, and extravasate into the parenchyma. Furthermore, these molecules result in homotypic melanoma cell adhesion and emboli formation.

Regarding variability of antigens on endothelial cells in different organs, depending on those shared with melanoma cells, melanoma has site-specific metastatic characteristics.

Local invasion: Expression of melanoma cell adhesion molecule MUC18 in melanoma is associated with activation of matrix metalloproteinases, leading to degradation of the basement membrane. Furthermore, expression of cadherins in melanoma cells is responsible for adhesion of malignant cells to keratinocytes and fibroblasts.

Vasculogenic mimicry: Expression of VE-cadherin in melanoma causes formation of capillary-like structures in tumor.

Angiogenesis and lymphangiogenesis: This tumor is highly angiogenic and lymphangiogenic, attributed to inflammatory molecules secreted by both malignant cells and the microenvironment.

Seed and soil hypothesis: Genetically unstable melanoma cells (seed) find the appropriate organ microenvironment (soil).

Premetastatic niche: Tumor produced-soluble molecules and bone marrow-derived cells result in melanoma cell proliferation, invasion, and metastasis via promoting formation of the metastatic microenvironment.

Stemness: Melanoma cells can act as stem cells resulting in repopulation of new tumors.

Parallel vs. linear progression: There is two models of metastasis in melanoma; one parallel to primary tumor progression and another one, metastasis from metastasis.

Immunogenicity: Although melanoma is very immunogenic, it evade cytotoxicity via some methods.

Transcriptional regulation: The progression of melanoma from the radial growth phase to the vertical one is attributed to changes in the gene transcription factors.

Mutation: Melanoma has the highest mutation rate in comparison with other malignancies.

Complementary: With understanding the mechanisms underlying aggressive manner of melanoma, we can successfully treat this tumor with target-targeting weapons.

\section{The efficacy of nalfurafine hydrochloride in the treat- ment of pruritus}

Studies have shown that the $\mu$ - and $\kappa$-opioid receptors play a great role in the regulation of pruritus in the central nervous system; on the other hand, the epidermal keratinocytes also express these receptors [5]. Nalfurafine hydrochloride is a potent and selective nonpeptide agonist of $\kappa$-opioid receptors with tyrosine-glycine moiety for endogenous opioid peptides, which was constructed in 1998 by a Japanese group. It appears that this agent exerts its antipruritic activity through the $\mathrm{k}$ receptors in the epidermis. In this study, Inui reviewed the efficacy of this drug in the management of pruritus induced by hemodialysis and other factors:

Hemodialysis-induced pruritus: Its exact pathophysiology is not well-understood. The probable roles of secondary hyperparathyroidism, histamine, mast cell-released tryptase, skin barrier dysfunction-induced increased epidermal neuron fibers, and the upper neuron system have been suggested in the pathogenesis of this kind of pruritus. The management of pruritus in patients under hemodialysis is a big challenge. Oral agents such as gabapentin, and thalidomide, topical agents such as endocannabinoid, capsaicin and tacrolimus, and ultraviolet B therapy have been administered with some success in managing of this kind of resistant pruritus. Different studies have shown the effectiveness and safety of nalfurafine in treating hemodialysis-induced pruritus.

Atopic dermatitis: As a causative factor in atopic dermatitisinduced pruritus, down-regulated expression of epidermal $\kappa$-opioid receptors has been suggested. It appears that nalfurafine can be effective in treating pruritus in patients with atopic dermatitis.

Psoriasis: Pruritus has been reported in some cases of psoriasis. It appears that the epidermal expression of $\kappa$-opioid receptor and dynorphin A is significantly decreased in these cases. Hence, nalfurafine can be useful in managing this kind of pruritus.

Cholestasis: Study on rats has revealed the potential effectiveness 
of nalfurafine in treating cholestasis-induced pruritus.

Drug-induced pruritus: The effectiveness of nalfurafine has been demonstrated in chloroquine-induced pruritus.

Commentary: Nalfurafine hydrochloride can be a good option for treating recalcitrant pruritus.

\section{References}

1. Brauer JA, Gordon Spratt EA, Geronemus RG (2014)Laser therapy in the treatment of connective tissue diseases: a review. Dermatol Surg 40: 1-13.[Crossref]
2. Hession MT, Graber EM (2015) Atrophic acne scarring: a review of treatment options. $J$ Clin Aesthet Dermatol 8: 50-58.[Crossref]

3. Sharquie KE, Noaimi AA (2014) Podophyllin $25 \%$ as alternative effective topical therapy for keratoacanthoma. Global Dermatol 1: 21-23.

4. Braeuer RR, Watson IR, Wu CJ, Mobley AK, Kamiya T, et al. (2014) Why is melanoma so metastatic? Pigment Cell Melanoma Res 27: 19-36.[Crossref]

5. Inui S (2015) Nalfurafine hydrochloride to treat pruritus: a review. Clin Cosmet Investig Dermatol 8: 249-255.[Crossref]

Copyright: $@$ 2016 Bagherani N. This is an open-access article distributed under the terms of the Creative Commons Attribution License, which permits unrestricted use, distribution, and reproduction in any medium, provided the original author and source are credited. 